Case Report

\title{
ANOMALOUS COM MUNICATION BETWEEN RIGHT INTERNAL ILIAC VEIN AND LEFT COM MON ILIAC VEIN - A CASE REPORT
}

\author{
Huban Thomas $\mathbf{R}^{1}$, , Prakashbabu $\mathrm{B}^{2}{ }^{2} \&$ Radhakrishnan $\mathrm{P}^{3}$ \\ ${ }^{1}$ Senior Lecturer, ${ }^{2}$ Associate Professor, ${ }^{3}$ P.G. Student, Department of Anatomy, Kasturba M edical College, \\ Manipal University, Manipal - 576 104. Karnataka, India. \\ Correspondence : \\ Huban Thomas R. \\ Department of Anatomy, Kasturba Medical College, Manipal University, Manipal - 576 104, Karnataka, India. \\ Mobile : +919844343546 E-mail : huban_anatomy@yahoo.co.in
}

\begin{abstract}
:
Inferior vena cava (IVC) is formed by the union of the common iliac veins anterior to the body of the fifth lumbar vertebra, a little to its right side. It conveys blood to the right atrium from all the structures below the diaphragm. During routine educational dissection for medical undergraduates, we have come across a case of an anomalous communication between right internal iliac vein and left common iliac vein and a variation in the formation of inferior vena cava in a 55 -year-old male cadaver. Due to its complex embryogenesis and relationship with other abdominal and thoracic structures, IVC may develop abnormally. These anatomical variations are often clinically silent and discovered incidentally. Knowledge of these variations may be helpful to clinicians and anatomists during surgical exploration, atypical clinical presentations and cadaveric findings.
\end{abstract}

Keywords: Inferior vena cava, common iliac veins, internal iliac vein

\section{Introduction:}

The internal iliac vein (hypogastric vein) begins near the upper part of the greater sciatic foramen, passes upward behind and slightly medial to the Internal iliac artery and, at the brim of the pelvis, joins with the external iliac vein to form the common iliac vein.

The common iliac veins are formed by the union of external and internal iliac veins. The left and right common iliac veins come to gether in the abdomen at the level of the fifth lumbar vertebra, forming the inferior vena cava. They drain blood from the pelvis and lower limbs.

The inferior vena cava (IVC) is the large vein that carries deoxygenated blood from the lower half of the body into the right atrium of the heart. It is formed by the union of the common iliac veins anterior to the body of the fifth lumbar

\section{Access this article online} Quick Response Code

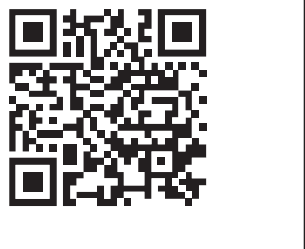
vertebra, a little to its right side. It is posterior to the abdominal cavity and runs along the right side of the vertebral column (i.e. it is a retroperitoneal structure). It enters the right atrium at the lower right, back side of the heart.

\section{Case Presentation :}

During routine educational dissection for medical undergraduates, we have come across a case of an anomalous communication between right internal iliac vein and left common iliac vein in a 55-year-old male cadaver. Formation of the IVC is also contributed by this anomalous communication between right internal iliac vein and left common iliac vein. Due to its complex embryogenesis and relationship with other abdominal and thoracic structures, IVC may develop abnormally.

\section{Discussion :}

Anatomical variation of the inferior vena cava occurs in 0.4-4\% of the population ${ }^{1}$.

Anomalies of the inferior vena cava and renal veins occur infrequently but if unidentified can lead to significant morbidity during surgical exploration ${ }^{2}$. Embryogenesis of the IVC is a complicated process involving development, regression, anastomosis and replacement of three pairs of venous channels: posterior cardinal, subcardinal and supracardinal veins ${ }^{3}$. 


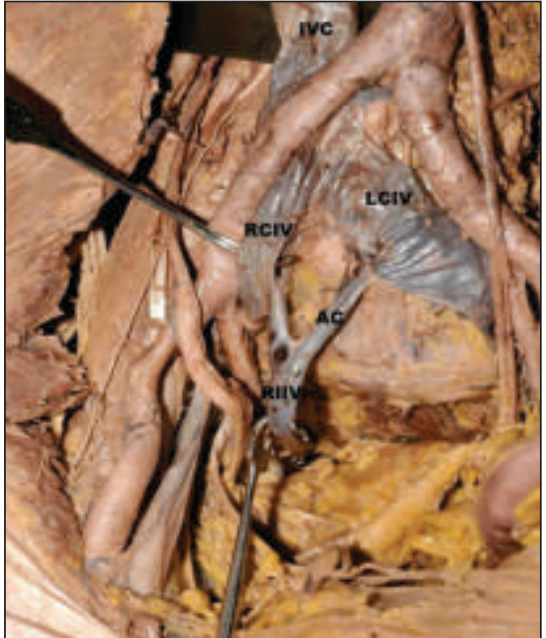

Figure : Superior view of the male pelvis

IVC - inferior vena cava, RCIV - right common iliac vein, LCIV - left common iliac vein. RIIV - right internal iliac vein, $A C$ - anomalous communication between right internal iliac vein and left common iliac vein.

The normal IVC converts to unilateral right sided system consisting of four segments, namely of hepatic, prerenal, renal and post renal segments. The hepatic IVC develops by coalescence of hepatic sinusoids, which are derived from the vitelline vein. The right sub cardinal vein develops into the suprarenal segment of IVC thus forming an anastomosis with the hepatic segment. The renal segment develops from anastomoses between the sub cardinal and

\section{References:}

1 Mayo J, Gray R, Louis ES, Grossman H, Loughlin M, Wise D. Anomalies of inferior vena cava. American Journal of Roentgenology1983; 140: 339-45.

2. Mathews R, Smith PA, Fishman EK, Marshall FF. Anomalies of the inferior vena cava and renal veins: embryologic and surgical considerations. Urology 1999; 53(5):873-880.

3. Artico D, Lorenzini P, M ancini P, Gobbi S, Carloia V. David Radiological evidence of anatomical variation of the inferior vena cava: Report of two cases. Surgical and Radiolgical Anatomy 2004; 26: 153-156.

4. Alicioglu B, Kaplan M, Ege T. Absence of infra renal Inferior vena cava is not a congenital abnormality. BratisI Lek Listy 2009; 110 (5): 304- 306.

5. Evans JC, Earis J, Curtis J. Thrombosed double inferior vena cava mimicking paraortic lymphadenopathy. The British Journal of Radiology2001; 74: 192-194.

6. Sénécail $B$, Lefevre $C$, Person $H$, Meriot P. Radiologic anatomy of duplication of the inferior vena cava: A trap in abdominal imaging. A report of 8 cases. Surgical and Radiologic Anatomy 1987; 9: 151-157.

7. Krishnamurthy A, Nayak SR, Khan S, Prabhu LV, Pai M M , Vadgaonkar R. Anomalous channels draining the internal iliac veins: embryological and clinical significance. Romanian Journal of Morphology and Embryology2007; 48(1):71-73. supra cardinal veins. The post renal IVC is formed by confluence of the common iliac veins, the caudal extremity of the right post cardinal vein, the right post cardinal-supra cardinal anastomosis, a part of the right supracardinal vein, and the right supra cardinal- sub cardinal anastomosis ${ }^{4}$.The aberrant development of these venous systems, for unknown reasons, causes anomalies of the IVC system ${ }^{3}$.

Most of these variations are found via radiology or during post-mortem, dissection of cadavers in university anatomy classes, their identification is important in the clinical realm because it may reduce misdiagnoses ${ }^{5}$. It has been reported that these variants can be confused with lymphadenopathy, aortic aneurysm, and retroperitoneal cysts, which often leads to unnecessary interventions ${ }^{6}$.

\section{Conclusion :}

A high index of suspicion on the part of the surgeon is required to prevent inadvertent injury to these anomalous veins and to avoid significant hemorrhage during retroperitoneal surgery. Preoperative assessment and intra operative awareness are important to prevent unexpected venous injuries ${ }^{7}$.Anomalous communication between right internal iliac vein and left common iliac vein is not been reported in the literature. 